\title{
The 6th Congress of the International Society for Applied Phycology, ISAP 2017, Nantes, France
}

\author{
Pascal Jaouen $^{1} \cdot$ Jean-Paul Cadoret $^{2} \cdot$ Valeria Montalescot $^{3}$ \\ Published online: 2 October 2018 \\ (C) Springer Nature B.V. 2018
}

The Executive Committee of the International Society for Applied Phycology trusted the city of Nantes, France, to organize its 6th Meeting in June 2017. More precisely, the University of Nantes and the company Greensea were chosen to co-chair this event. With a strong academic and private involvement, Western France is very active in the field of marine biotechnology and in particular in phycology. Thank the help of a dynamic Local Organizing Committee (AlgoSource, Ifremer, Cluster "Pôle Mer Bretagne Atlantique," Atlanpole) the congress was a success. The local organizing committee thanks the Society and in particular, Roberto de Philippis, for trusting the French team to organize this triennial event. With 560 participants, 150 talks, 250 posters, 45 countries, 14 exhibitors, and 75 participants to the B2B organized by AlgoSource, the congress proved the dynamics of that field.

Several considerations had to be taken into account. An equilibrium had to be found between microalgae and macroalgae. This was a rather difficult task considering concurrent and well-established event for seaweeds in particular. A way between upstream and downstream works had to be found. A subtle balance toward fundamental research in an applied congress. The evaluation and the choice for a

Jean-Paul Cadoret

jeanpaulcadoret@greensea.fr; https://isap2017.sciencesconf.org/

Pascal Jaouen

https://isap2017.sciencesconf.org/

Valeria Montalescot

https://isap2017.sciencesconf.org/

1 Université Nantes, GEPEA, CNRS, 37 Bd de l'Université BP 406, 44602 Saint-Nazaire, France

2 Greensea Company, Promenade du sergeant Navarro, 34140 Meze, France

3 Université Nantes, GEPEA, CRTT, 37 Bd de l'Université BP 406, 44602 Saint-Nazaire Cedex, France repartition between oral and poster presentation was a difficult task. This has been achieved thanks to a panel of 46 reviewers from a selected International Advisory Committee.

Such congress cannot be organized without a reliable support. The University of Nantes provided a strong support in many strategic points, (Financial warranty, Communication strategy, Logistics...). The involvement of our volunteers, mainly students. More than 40 sponsors accepted to help and thus showed interested and participated in building the international visibility.

This was the first time that the ISAP congress decided to favor meetings of industries in organizing a BtoB event that attracted 75 registrations.

As a result, this congress being at first at scientific meeting, we received more than 400 abstracts $(30 \%$ on seaweeds and $70 \%$ on microalgae) providing a scope of the international applied research in this field. If the topics and sessions were logically close to the one chosen in the previous meetings, the achievements show a rising maturity. A maturity in the tools now provided to the research as well as to the industry. As examples, the biorefinery platform (AlgoSolis, AlgaePark...) a maturity in the concepts flourishing and inspiring (high-level "omics" tools).

The congress was launched with an excellent opening "Tara Oceans: Eco-Systems Biology at Planetary Scale" from Chris Bowler that reminds us how immense is the potential of algae diversity and how little we know about it.

The topics were arbitrarily divided in four domains: Process, Industry, Biology, and Applications. With $31 \%$ of the papers in Applications, 29\% in Biology, $11 \%$ in Industry, and $28 \%$ in Process.

There were eight captivating plenary presentations representing the four major domains on both Seaweeds and Microalgae:

Arthur Grossman-The discovery of novel proteins required for photosynthetic function using the model green alga Chlamydomonas reinhardtii. 
Claire Gachon-Novel strategies for preventing diseases in cultivated seaweeds.

Makoto Watanabe-Next-generation health functions of microalgae.

Maria Barbosa - Toward industrial products from microalgae.

Vitor Verdelho-Contributions for the nomenclature of microalgae production systems.

Anne Hurtado-Seaweed extracts as biostimulant in Kappaphycus cultivation.

Vitalia Henriquez-Microalgae genetic engineering tools for the production of high-value compounds.

Claudia Grewe-Industrial application of microalgae in food, feed and cosmetics - current status, and perspectives.

With the aim of avoiding several parallel sessions, the Organizing Committee decided to limit the talks to 150 split into two parallel sessions. The sessions in Biology covered mainly topics related to omics tools applied to strain identification, development, and selection to physiology, carbon metabolism, and photoacclimation, and to the identification and production of bioactive molecules.

The Process session offered insights in algae cultivation monitoring, metabolic orientation, algae cultivation in effluents, harvesting, and multi-step valorization of the biomass through biorefinery examples.

A wide variety of topics were discussed during the Application sessions covering aquaculture, nutraceuticals, health, food, cosmetics, green chemistry, or agriculture with talks on algae compounds as growth promoters, biostimulants, and fertilizers; algae digestibility and their potential as functional food; immune-modulating and prophylactic activities from algae extracts, or bioplastic production, for example.

The Industry sessions focused on large-scale production and biorefinery, legislation aspects as novel food and Nagoya Protocol, finalized products, and market tendencies for the next years.

The young phycologists were also awarded:

\section{Oral Presentations}

\section{Seaweeds}

1. Miriam Bernard (Roscoff-Univ. Paris 6-France): qPCR-based detection of a filamentous brown algal endophyte in Saccharina latissima wild populations and kelp farms

2. Anne-Sophie Burlot (Université Bretagne Sud - France): An enzymatic extract of the proliferative red seaweed Solieria chordalis reduces the virulence of Pseudomonas aeruginosa and stimulates the immune system of Caenorhabditis elegans.

3. Dara Kirke (Martin Ryan Institute, NUI-GalwayIreland): Single and interactive effects of PAR and UV on low molecular weight phlorotannin profiles in Fucus vesiculosus using mass spectrometry.

\section{Microalgae}

1. Kohei Yoneda (University of Tsukuba_Japan): A discovery of the novel major lipid droplet protein in marine diatom Phaeodactylum tricornutum

2. Maria Cuello (National Technological UniversityArgentina; Murdoch University-Australia): Culture of microalgae in mixed wastewater streams.

3. Zachor Adler-Agmon (Ben-Gurion University-Israel): Nitrogen starvation-induced expression of proteins in Phaeodactylum tricornutum.

In addition to the scientific program the Congress also hosted different meetings of the Atlantic Microalgae Consortium General Assembly; meeting of the GDRI WONDER « World Oilalg Network for Design of Processes and Strains for Elaboration of Renewable energy from microalgae » (Murdoch University, Australia; Tsukuba University, Japan; Univ. California UCLA, USA; GEPEACNRS-Univ. Nantes, France); " Seaweed at Stake »: Stakeholder meeting catalyzing future collaboration (Organized by MAFOR/MARFORSTAKE and Phycomorph) and a public conference for the Nantes Citizens: "Les algues: de la Biodiversité Marine à la conquête spatiale » by Colomban de Vargas (CNRS Station Biologique Roscoff, Tara Expeditions) and Christophe Lasseur (European Space Agency, Melissa Program).

Of course, the International Society for Applied Phycology (ISAP) General Assembly took place, which formalized the setting up of the new ISAP Executive Committee, the confirmation of Céline Rebours as President and Roberto de Philippis as outgoing President for the following 3-year period. Since then, the election of Qiang Hu as Vice-President has been confirmed.

During the social event, surrounded by the original universe of "Les Machines de l'Ile" at the crossroads of Jules Verne's "invented worlds," the mechanical universe of Leonardo da Vinci, and of Nantes' industrial history, four eminent society members were awarded for their continuous and outstanding contributions to the field of Applied Phycology: Avigad Vonshak, John Benemann, Susan Blackburn, and Roberto de Philippis.

With the highest numbers of attendees since the beginning of the Society (Almeria more than 300, Galway 430, Halifax 320 , Sydney 285, Nantes 560) and with more than 40 sponsors, 38 supports, and 14 exhibitors, the 6th edition of ISAP congress has successfully reflected the dynamism of the applied phycology community. 